\title{
Assessing Measurement Distances for OTA Testing of Massive MIMO Base Station at $28 \mathrm{GHz}$
}

\author{
Pekka Kyösti ${ }^{12}$, Wei Fan ${ }^{3}$, Jukka Kyröläinen ${ }^{1}$, \\ ${ }^{1}$ Keysight Technologies Finland oy, Oulu, Finland, pekka.kyosti@keysight.com \\ ${ }^{2}$ University of Oulu, CWC, Oulu, Finland, pekka.kyosti@ee.oulu.fi \\ ${ }^{3}$ Aalborg University, Antennas, Propagation and Radio Networking section \\ at the Department of Electronic Systems, Faculty of Engineering and Science, Denmark, wfa@es.aau.dk
}

\begin{abstract}
This paper discusses physical dimensions for a multi probe anechoic chamber based (MPAC) over-the-air (OTA) setup aiming for base station (BS) testing. The target frequency of the simulated massive multiple-input-multiple-output (MIMO) BS arrays is $28 \mathrm{GHz}$. The assessment is performed with two metrics. The first metric is a new power metric based on assumptions of a code book of fixed beams and planar waves. The second one is the multi-user (MU) MIMO sum rate capacity. The intention is to evaluate physical dimensions in metres with respect to different BS array sizes. Simulation results indicate that OTA performance of a BS array with maximum dimension of $0.15 \mathrm{~m}$ could be measured with a setup having measurement distance of approximately $1 \mathrm{~m}$.
\end{abstract}

Index Terms-Massive MIMO base station; performance evaluation; over the air testing; multi-probe anechoic chamber setup.

\section{INTRODUCTION}

Active research and development is ongoing for a new telecommunication system with working title $5 \mathrm{G}$. The $5 \mathrm{G}$ concept contains many requirements like, e.g., ultra reliable communication, massive machine type communication, and mobile broadband [1]. Mobile broadband offers high bit rates with the freedom of mobility. Higher data rates for mobile broadband require wider bandwidth, which is available at higher frequencies. Further, massive MIMO antenna systems at base stations enable serving multiple users at same frequency band and also help to compensate higher path loss, inherent to high frequency, by array gains [2]. For the first pre-5G trials $28 \mathrm{GHz}$ frequency area has been announced by a number of operators and vendors.

The current BS testing is typically performed in a conducted manner. Antenna array characteristics are known or measured separately and many air interface testings with or without fading channel emulation are carried out by connecting test devices to BS antenna ports with coaxial cables. However, according to the views of the industry this approach is foreseen to become obsolete. Future BSs operating at higher frequencies, e.g., at $28 \mathrm{GHz}$ are predicted to be such highly integrated units, that no antenna (radio frequency) connectors are available. Not even for testing purposes. Thus the connectivity and the access to $\mathrm{BS}$ has to be achieved through antennas - in the radiating mode.
Over-the-air testing for small devices has been under research for many years and is already in a mature phase. The MPAC method [3] has been standardized in CTIA [4] and 3 GPP [5]. However, in the latter one there are still investigations ongoing. There are some key differences between typical radio channel conditions on user equipment (UE) and BSs, as discussed in [6]. Compared to UEs a BS is normally elevated from the ground level and farther from any obstacles, thus resulting in smaller per path angular spread. Further, BSs, especially for small cells, are sectored, i.e. intended to serve only a sector of angles. On the other hand, for UE testing 2-dimensional (horizontal) power angular distribution is considered sufficient in [4], [5] while for BS testing the radio environment has to be modelled in 3-dimensions. Thus a sectored 3D configuration of probes is suitable for the radiated testing of BSs with the MPAC method.

In this paper we continue the work introduced in [6], but now with focus on $28 \mathrm{GHz}$ frequency band. Device under test (DUT) is a base station with planar antenna array. At $28 \mathrm{GHz}$ even massive MIMO arrays are relatively small, from only some to few tens of centimetres. The purpose is to assess the minimum measurement distance, which directly indicates the overall size and is proportional to the cost of the test setup. We propose a new metric for the evaluation, based on power loss with a code book of fixed beams.

\section{MPAC SETUP}

A sectored MPAC test setup contains an anechoic chamber, a number dual polarized probes in a sector configuration, a fading emulator and a number of UEs or UE emulators, as illustrated in Fig 1. In total $N$ probes and an array of $M$ BS elements are placed in a coordinate system as shown in Fig 2. The origin is in the centre of BS array and each probe has distance $R$ to the origin. The BS is assumed to have rectangular planar array with the broad side pointing to centre of the probe configuration. More details are given in [6].

\section{MEtriCs FOR MEASUREMENT DistanCE}

\section{A. Fixed beam power loss}

This is a new metric to evaluate the impact of shortening measurement distance $R$ below Fraunhofer distance. Here 


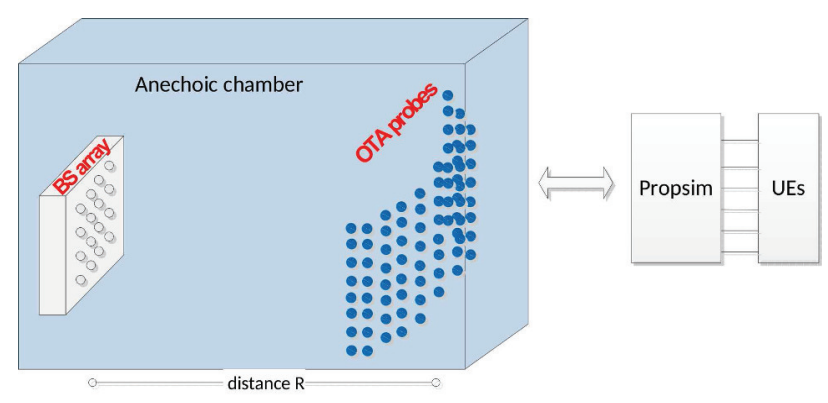

Fig. 1. Sketch of sectored MPAC setup.

$\mathrm{R}=0.4 \mathrm{~m}$, azim $=120 \mathrm{deg}$, elev $=60 \mathrm{deg}$, DUT diagonal $=0.14 \mathrm{~m}$

Chamber (tight) dimensions $=0.4 \times 0.7 \times 0.4$ meters

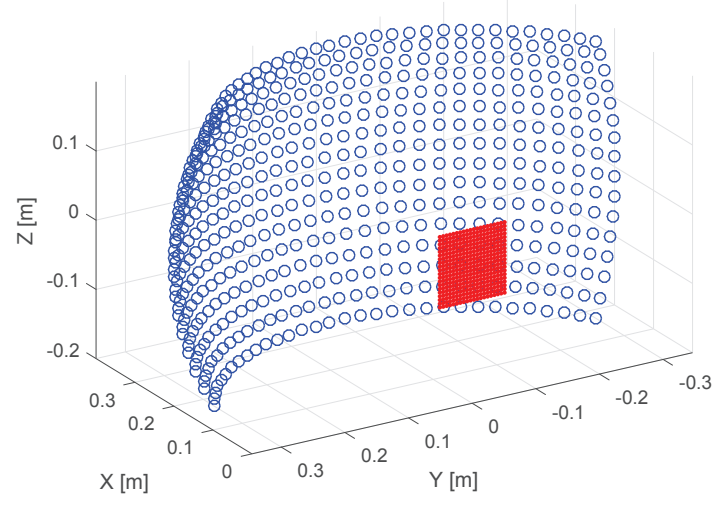

Fig. 2. The setup of probes (blue) and DUT array (red).

we assume a communication system with fixed beams, i.e. with a discrete code book of BS antenna weights. The BS array can at least partly utilize analog combining of elements (analog beamforming) to compose beams to a pre-defined set of directions. This is the expected mode of massive MIMO BSs in so called pre-5G systems [7]. It is further assumed the BS array is well calibrated and the fixed main beams are targeted to certain directions, i.e. for receiving planar wavefronts. Now with limited $R$ the probes are near and the BS array does experience spherical wavefronts instead of planar as illustrated in Fig 3.

The metric is derived as follows. Probe locations are defined

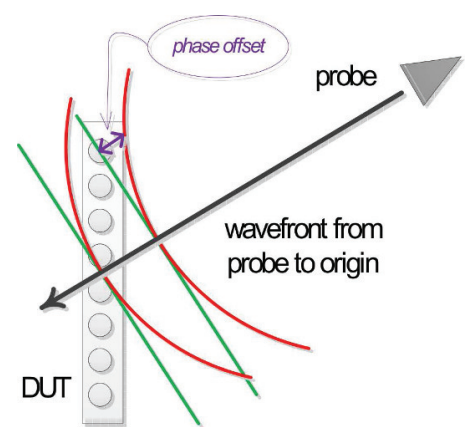

Fig. 3. Definition of phase deviation between a planar and a spherical waves across the DUT. by position vectors $\bar{a}_{n}$ and DUT element locations with $\bar{b}_{m}$. Phase offset $\alpha_{n m}$ is calculated for a spherical wave from probe $n$ to DUT element $m$ with respect to the phase in the origin

$$
\alpha_{n m}=\left(\left\|\bar{a}_{n}-\bar{b}_{m}\right\|-R\right) \frac{2 \pi}{\lambda}
$$

where $\|\cdot\|$ denotes the vector norm operation and $\lambda$ is the wavelength on carrier centre frequency. Similarly phase offset for a planar wavefront in direction of $n$th probe is

$$
\beta_{n m}=\frac{-\bar{a}_{n}}{\left\|\bar{a}_{n}\right\|} \cdot \bar{b}_{m} \frac{2 \pi}{\lambda},
$$

where $\cdot$ denotes the dot product operation.

It is assumed that with a fixed beams system the planar waves from the main direction of a beam results to coherent summation of signals from BS antennas. In the case of spherical waves the summation is partly incoherent and results to a loss of received power and consequently to a reduction of signal to noise power ratio. The loss for direction of $n$th probe can be calculated utilizing cosine function as follows

$$
Q_{n}=\left|\frac{1}{M} \sum_{m=1}^{M} \cos \left(\beta_{n m}-\alpha_{n m}\right)\right|^{2}
$$

Further, the average fixed beam power loss is taken over all $N$ probe locations

$$
Q_{a v}=\frac{1}{N} \sum_{N=1}^{N} Q_{n} .
$$

The idea of eq (3) and (4) is illustrated in the complex plane of Fig 4. On the left hand figure the blue circles denote phasors of a curved wave at each BS antenna element location and the red stars corresponding phasors of a planar wave, both from the same (probe) direction. In the right hand figure the phasors are multiplied with the weights that assume planar wave from the direction. The red phasors sum coherently resulting to $100 \%$ gain, while the blue phasors have some phase variation, summing up only to $95 \%$ gain. The fixed beam power loss is the ratio of these gain values.

An example of the fixed beam power loss distribution across beam directions is shown in Fig 5. In this case the BS is $20 \times$ 20 planar array and $R=0.9 \mathrm{~m}$. The phase error of spherical waves from the broad side of BS arrays is higher compared to other directions, as can be understood from Fig 3 and as shown also in [6]. This results to more severe power loss for directions close to the broad side, as can be observed from the example. Simulated probability distributions of average fixed beam power losses calculated with eq. (4) are introduced in Section IV-A.

Interestingly, it should be possible to develop this metric to an analytic formulation, that would save the numerical simulation of the values. Discrete probe location would be substituted by ranges of the azimuth and elevation sector. Similarly discrete DUT element locations could be replaced by ranges of $y$ and $z$ coordinates of the DUT array surface. The 

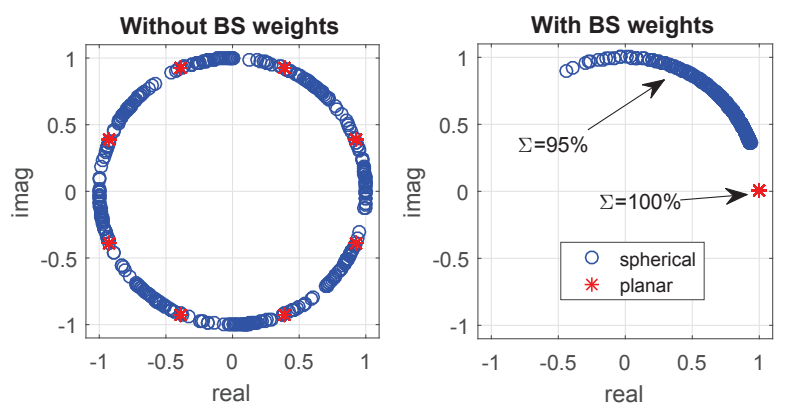

Fig. 4. Phasors at BS antennas without (left) and with (right) applying antenna weights for a fixed beam.

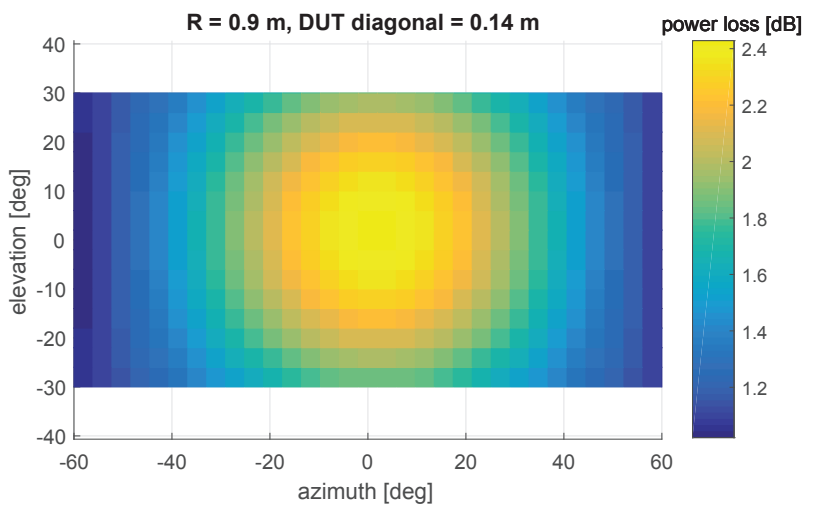

Fig. 5. Example of the fixed beam power loss in azimuth and elevation angles.

average power loss would be determined by definite integration over the ranges. We leave developing the analytical solution for future work.

As usual, it is difficult to claim any strict threshold for the metric. We try to relate the power loss to coverage, to make it more meaningful. Inevitably $28 \mathrm{GHz}$ BSs are targeted for very small cells. Assume the cell edge is in 25 metres. Further assuming the free space path loss formula we can approximate how many metres is lost from the full $(25 \mathrm{~m})$ coverage with certain fixed beam power loss values as depicted in Fig 6. For example a loss of $3 \mathrm{~dB}$ in the beamforming received power and a demand to keep the same SNR would shorten the link distance from $25 \mathrm{~m}$ to $17.5 \mathrm{~m}$, i.e. by $7.5 \mathrm{~m}$.

\section{B. MU-MIMO sum rate capacity}

While the fixed beam power loss defined in the previous section III-A assumed discrete set of element weighting, enabling at least partly analog beamforming, the MU-MIMO capacity here presupposes full freedom in combining elements of the BS array (full digital beamforming). Massive MIMO antenna arrays at BSs can be utilized for communicating with

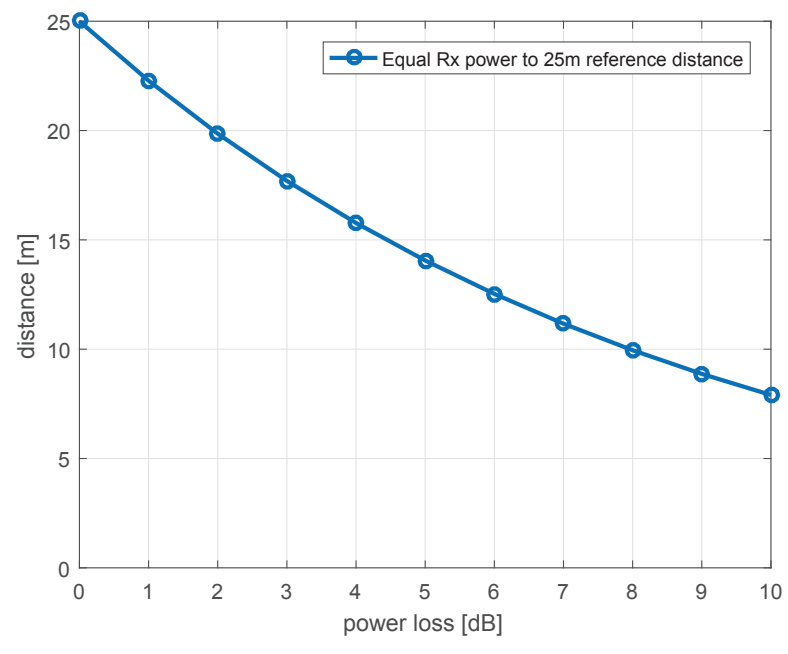

Fig. 6. Illustration of coverage shortening as a result of fixed beam power loss assuming the reference link distance $25 \mathrm{~m}$ and free space path loss.

a number of spatially separated UEs on same time-frequency resources. MU-MIMO sum rate capacity [8] is another metric to evaluate the impact of limited measurement distance $R$. In evaluations we take the distance $R=1000 \mathrm{~m}$ as the reference condition where all waves fronts from probes to DUT are practically planar.

The procedure to simulate sum rate capacities in sectored MPAC setup is described in [6]. We summarize it briefly here. Radio channel parameters and channel responses are generated with Keysight Geometric Channel Modelling Tool for each BS-UE link. The nominal azimuth and elevation angles at BS side (AoD and EoD) of each multi-path of each UE are mapped to the example configuration of probes, illustrated in Fig 2, as describe in [6]. The result is a composite channel transfer matrix $\mathbf{H}(t)$ for a sub-set of UEs and all BS elements. The channel matrix is normalized to have equal channel gain between UEs and overall unit gain over time samples.

Next, linear pre-coding vectors are determined for each UE of the sub-set by a sub-optimal linear pre-coding method, zero forcing [8]. The method necessitates full freedom to weight BS elements to null the interference between users. The matrix of pre-coding vectors is calculated as

$$
\mathbf{W}(t)=\mathbf{H}^{H}(t)\left(\mathbf{H}(t) \mathbf{H}^{H}(t)\right)^{-1}
$$

after which the rows $\mathbf{w}_{k}(t)$ of $\mathbf{W}(t)$ are normalized. Finally the sum rate capacity is [8]

$$
C(t)=\sum_{k=1}^{K} \log _{2}\left(1+\frac{\gamma}{K}\left|\mathbf{w}_{k}(t) \mathbf{h}_{k}(t)\right|^{2}\right)
$$

where the signal-to-noise ratio $\gamma=20 \mathrm{~dB}$ and $\mathbf{h}_{k}(t)$ are column vectors of $\mathbf{H}(t)$. 


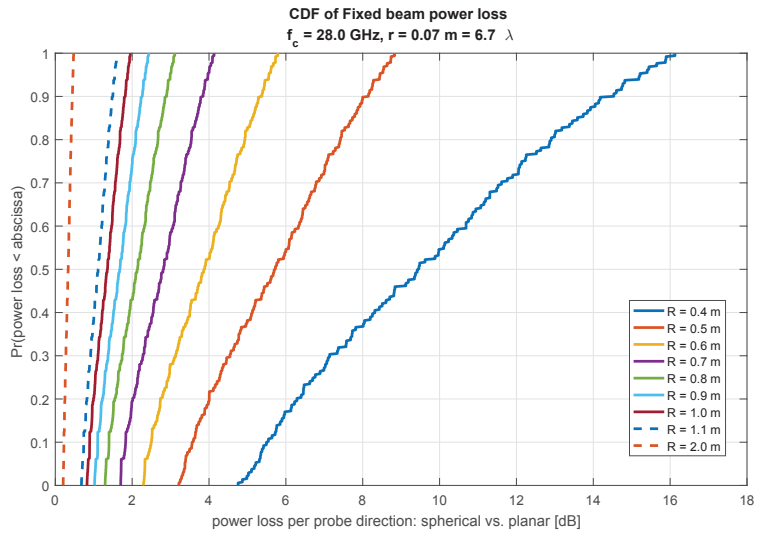

Fig. 7. CDFs of the fixed beam power loss with varying measurement distance $R$

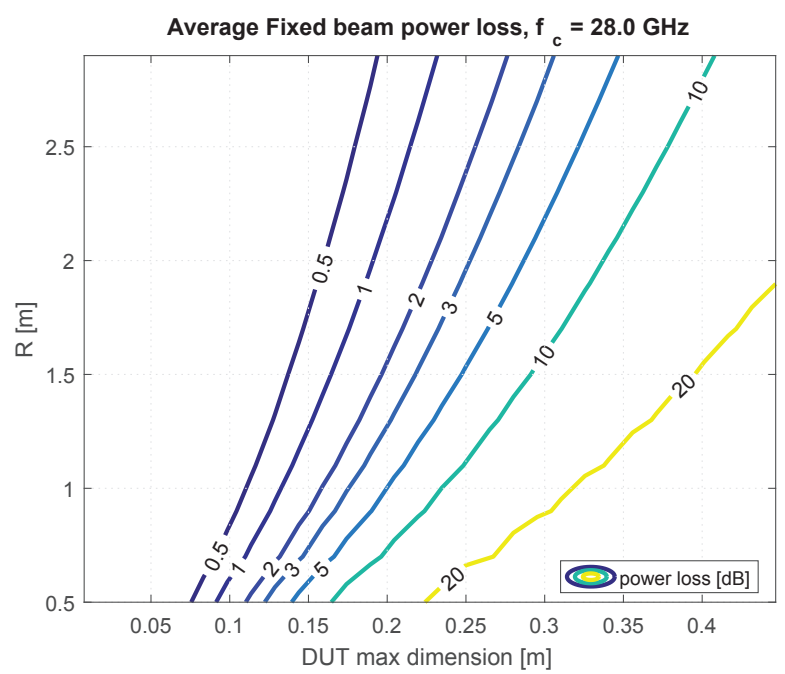

Fig. 8. Average fixed beam power loss as a function of measurement distance $R$ and BS arrays maximum dimension.

\section{Simulation RESUlts}

\section{A. Fixed beam power loss}

Cumulative distribution functions (CDF) of the fixed beam power loss are plotted in Fig 7 . The DUT is $20 \times 20$ planar array with $0.5 \lambda$ spacing between elements in vertical and horizontal dimensions. The maximum dimension of the array is $D \approx 13.4 \lambda \approx 0.144 \mathrm{~m}$.

Average fixed beam power losses as a function of measurement distance $R$ and DUT maximum dimension are shown in a contour plot in Fig 8. If we take, e.g., $2 \mathrm{~dB}$ loss as the acceptance criteria, we can approximate the relation between $D$ and $R$ with the linear function $R=14.46 D-1.1$. With the example BS array of $20 \times 20$ elements and $2 \mathrm{~dB}$ criteria the minimum $R$ would be $0.85 \mathrm{~m}$ and with the linear approximation slightly below $1 \mathrm{~m}$.

\section{B. Sum rate capacity}

The channel model selected for this evaluation is IMTAdvanced UMi scenario [9]. BS antenna is again the $20 \times 20$
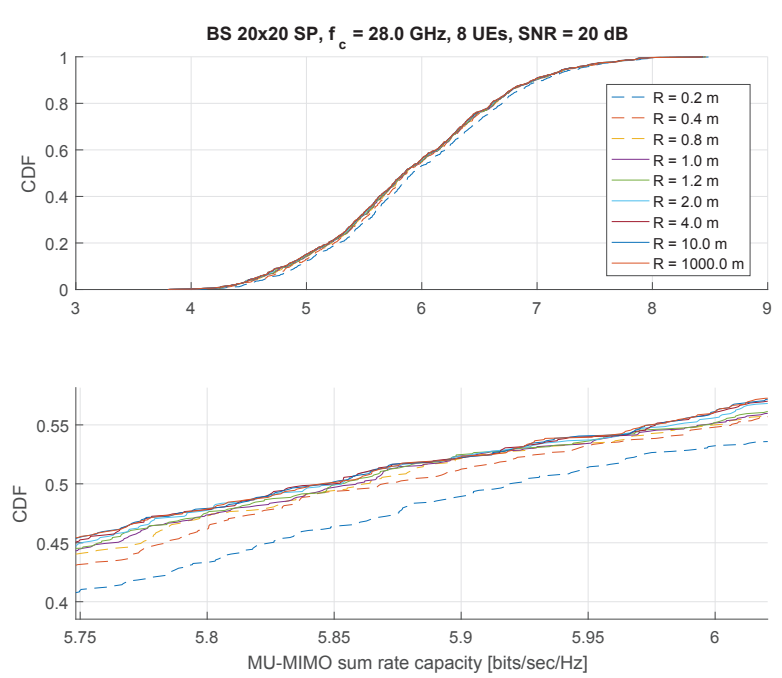

Fig. 9. CDFs of MU-MIMO sum rate capacities with different measurement distances $R$ (top) and a zoomed in view of it (bottom).

planar array with vertically polarized elements and half wavelength spacing. Sub-sets of $K=8 \mathrm{UEs}$ were picked randomly from the full set of 30 UEs. The simulation was repeated for 300 random UE sub-sets each with 1000 time samples and for nine distance $R=0.2,0.4,0.8,1,1.2,4,10,1000$ metres, where the last $1000 \mathrm{~m}$ case imitates the ideal case of $R=\infty$. For a single sub-set the cumulative distribution function (CDF) of the sum rate capacity is shown in Fig 9. Mean and standard deviations of sum rate capacities are depicted in Fig 10 with bar plots. Also the average deviation from the reference case of $R=1000 \mathrm{~m}$ is shown in Fig 10 .

We can observe from the CDF that only $R=0.2 \mathrm{~m}$ is clearly diverged from the set of curves. Further, the deviation curve indicates that the error is small, less than $3.5 \%$, in all cases and decreases rapidly with increasing $R$. When $R>1 \mathrm{~m}$ the deviation is below $1 \%$. Also can discovered that the utilized zero forcing method is highly adaptive, assuming full flexibility of BS antenna weights, and is not very sensitive to small measurement distances.

\section{CONCLUSIONS}

We presented two metrics to evaluate the minimum measurement in radiated testing of massive MIMO BSs. The fixed beam power loss metric, that assumes fixed beams operational mode by BSs, is expected to be a good evaluation metric to the test setup for BSs of the coming pre-5G systems. The MUMIMO sum rate capacity presumes full digital beamforming capability, that may not be a practical implementation for very large MIMO arrays. The fixed beam power loss indicates longer measurement distance requirement as the sum rate capacity metric. However, inferring stringent requirements with neither of the metrics is difficult. It is important to notice that the evaluated error metrics are related to the measurement 


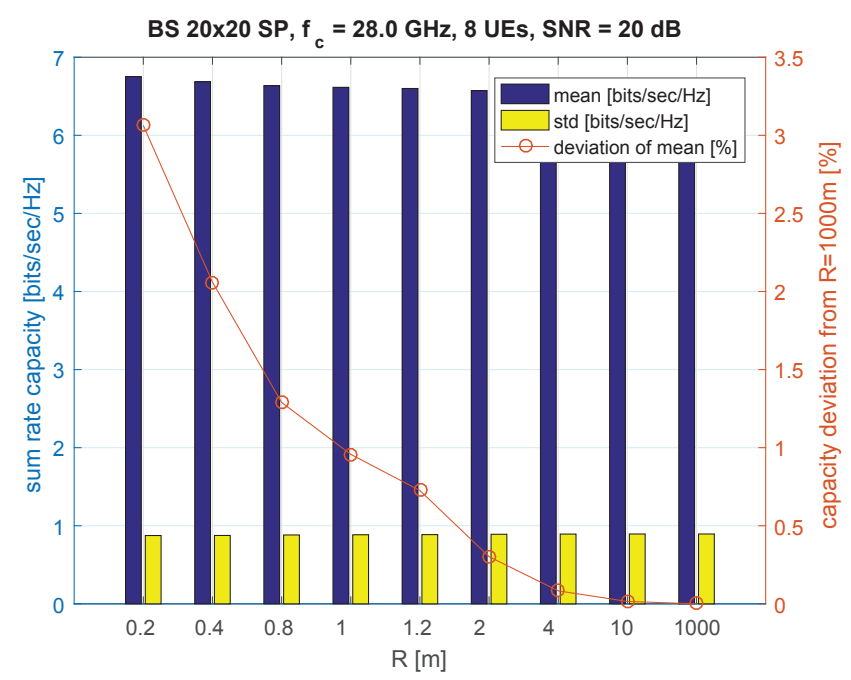

Fig. 10. Deviations of average MU-MIMO sum rate capacities with different measurement distances $R$.

distance only. Actual probe configurations, i.e. the numbers or the locations of probes, have not been considered in this work.

If the maximum accepted fixed beam power loss is set to $2 \mathrm{~dB}$ the relation of maximum DUT dimension $D$ and the minimum measurement distance $R$ can be approximated as $R=14.46 D-1.1$. With a large BS array of 400 elements $(20 \times 20)$ the requirement for $R$ would be slightly below $1 \mathrm{~m}$. Further, this would result to less than $1 \%$ deviation of the sum rate capacity.

\section{ACKNOWLEDGMENT}

The work of Dr. Wei Fan was supported by the Financial assistance from the Danish council for Independent Research under Grant DFF 6111-00525.

\section{REFERENCES}

[1] A. Osseiran, J. F. Monserrat, P. Marsch, M. Dohler, and T. Nakamura, Eds., $5 G$ Mobile and Wireless Communications Technology. Cambridge University Press, July 2016.

[2] E. G. Larsson, O. Edfors, F. Tufvesson, and T. L. Marzetta, "Massive mimo for next generation wireless systems," IEEE Communications Magazine, vol. 52, no. 2, pp. 186-195, February 2014.

[3] P. Kyösti, T. Jämsä, and J.-P. Nuutinen, "Channel modelling for multiprobe over-the-air MIMO testing," International Journal of Antennas and Propagation, vol. 2012, 2012.

[4] "Test Plan for 2x2 Downlink MIMO and Transmit Diversity Over-the-Air Performance," CTIA Certification, Tech. Rep. Version 1.0, August 2015.

[5] RP-160603, "Radiated performance requirements for the verification of multi-antenna reception of UEs," 3GPP, Tech. Rep., March 2016.

[6] P. Kyösti, W. Fan, G. F. Pedersen, and M. Latva-aho, "On dimensions of OTA setups for massive MIMO base stations radiated testing," IEEE Access, vol. PP, no. 99, pp. 1-1, 2016.

[7] "Air interface working group; verizon 5th generation radio access; physical layer procedures," Verizon 5G TF, Tech. Rep. TS V5G.213 v1.0 (2016-06), June 2016

[8] F. Kaltenberger, D. Gesbert, R. Knopp, and M. Kountouris, "Correlation and capacity of measured multi-user MIMO channels," in 2008 IEEE 19th International Symposium on Personal, Indoor and Mobile Radio Communications, August 2008, pp. 1-5.

[9] "Guidelines for evaluation of radio interface technologies for IMTAdvanced, ITU-R Report M.2135,” ITU-R, Tech. Rep., 2008. 